in vivo $36: 350-354(2022)$

doi:10.21873/invivo.12709

\title{
Surgical Techniques of Y-Sleeve Lobectomy in Patients With Primary Lung Cancer
}

\author{
TAKUMA TSUKIOKA, NOBUHIRO IZUMI, HIROAKI KOMATSU, \\ HIDETOSHI INOUE, RYUICHI ITO and NORITOSHI NISHIYAMA
}

Department of Thoracic Surgery, Osaka City University, Osaka, Japan

\begin{abstract}
Background/Aim: The directions of distal and proximal airway stumps were different in Y-sleeve lobectomy. This difference might make Y-sleeve lobectomy a difficult procedure. In this article, we present our surgical techniques and analyse short-term outcomes of Y-sleeve lobectomy. Patients and Methods: Right middle and lower, left lower, and left lower and lingular segment sleeve lobectomies are categorized in Y-sleeve lobectomy. We retrospectively investigated the clinical courses of 17 patients who underwent Y-sleeve lobectomy from January 2017 to December 2020. Results: No treatment-related deaths occurred. One patient developed a bronchopleural fistula. Four patients developed pneumonia and were cured by repeated bronchoscopies and antibiotic therapy. Three patients had retention of pleural effusion, and two had prolonged air leakage. One patient had empyema after prolonged air leakage and was cured by thoracic drainage and antibiotic therapy. Conclusion: A major complication was observed only in one patient. Y-sleeve lobectomy is a reliable surgical method to avoid pneumonectomy.
\end{abstract}

Tension (1) and calibre mismatch (2) at the anastomosis sites are important factors for airway healing after bronchial sleeve resection. The direction of the distal stump during bronchoplasty is almost identical to the original direction in many types of sleeve lobectomy. However, in some types of sleeve lobectomy, such as right middle and lower sleeve lobectomy, left lower sleeve lobectomy, and left lower and

This article is freely accessible online.

Correspondence to: Takuma Tsukioka, Department of Thoracic Surgery, Osaka City University, 1-4-3 Asahimachi, Abeno-ku, Osaka 545-8585, Japan. Tel: +81 666453841, Fax: +81 666466057, e-mail:m1156870@med.osaka-cu.ac.jp

Key Words: Y-sleeve lobectomy, right middle and lower sleeve lobectomy, left lower sleeve lobectomy, left lower and lingular segment sleeve lobectomy, bronchoplasty. lingular segment sleeve lobectomy, the direction of the distal stumps change greatly after airway reconstruction. Maurizi et al. (3) defined right middle and lower sleeve lobectomy and left lower sleeve lobectomy as 'Y-sleeve lobectomy'. The change in the direction of the airway may increase the complexity of bronchoplasty after Y-sleeve lobectomy compared to usual sleeve lobectomy. In the present study, we analysed our surgical techniques and short-term outcomes of sleeve lobectomy with a change in the direction of the distal airway stump.

\section{Patients and Methods}

In this study, we defined Y-sleeve lobectomy as sleeve resection requiring reconstruction with a change in the direction of the distal airway. Thus, Y-sleeve lobectomy included right middle and lower sleeve lobectomy, left lower sleeve lobectomy, and left lower and lingular segment sleeve lobectomy. We retrospectively investigated the clinical courses of 17 patients who had undergone Y-sleeve lobectomy at our Institute from January 2017 to December 2020. Before surgery, all patients provided informed consent for the use of their examination outcomes and data in clinical studies. The local institutional ethics committee approved this study (approval no. 4403; approval date, 3 October 2019).

Mediastinal lymph nodes with a short axis of $>10 \mathrm{~mm}$ on computed tomography (CT) were diagnosed as clinically positive for metastasis. Our criteria for surgical resection were the absence of distant metastasis, no cancer cell-positive pleural or pericardial effusion, no N2 disease at two or more mediastinal levels, no bulky $\mathrm{N} 2$ disease, no N3 disease, and a predicted postoperative percent vital capacity of $>40 \%$. Patients with T4 lung cancer with N0 or N1 nodal extension and tumours that could be completely removed were considered candidates for surgery. Sleeve lobectomy was performed if anatomically appropriate. Bronchial stumps were usually confirmed as free of cancer cell infiltration by intraoperative pathological examination.

The surgical approach was posterolateral thoracotomy in all patients. On the right side, a middle and lover sleeve bilobectomy was performed for lung cancer or metastatic lymph node in the bronchus intermedius which extended to origin of the upper lobe bronchus. On the left side, a left lower sleeve lobectomy was performed for lung cancer or metastatic lymph node in the left lower bronchus which extended to origin of the upper lobe bronchus. A left lower and lingular segment sleeve lobectomy was performed for lung 
a

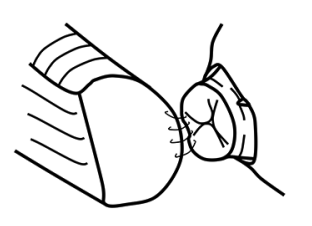

b

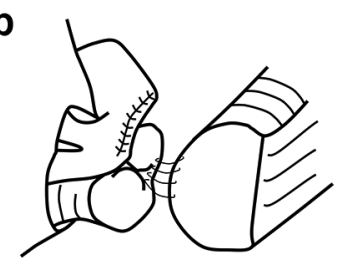

c

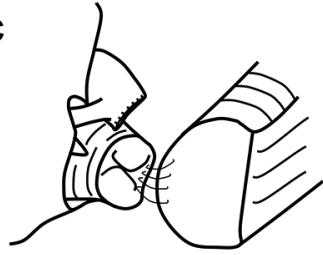

Figure 1. Four to five continuous 4-0 absorbable monofilament sutures were placed in the ventral portion of the anastomosis after (a) right middle and lower sleeve lobectomy, (b) left lower sleeve lobectomy, and (c) left lower and lingular segment sleeve lobectomy. After fixation of the ventral portion, the direction of the distal airway was about 90 degrees different from the direction of the proximal airway.

cancer or metastatic lymph node in left lower bronchus which extended to a distal portion of segmental bifurcation of the upper lobe bronchus. In this method, the upper and lingular segments were separated, followed by left upper segmental and main bronchi were divided at appropriate levels. Subcarinal and ipsilateral lower paratracheal lymph nodes dissection was performed in all patients. On the left side, the left main bronchus was divided from the pericardium. A mobilization of the left main bronchus allowed us to complete the bronchoplasty more easily. An anastomotic technique was same in all types of Y-sleeve lobectomy. Four to five continuous 4-0 absorbable monofilament sutures were performed on the ventral portion of the anastomosis. After the fixation of the ventral portion, the direction of the distal airway was about 90 degrees different from the direction of the proximal airway in all types of Y-sleeve lobectomy (Figure 1a-c). Interrupted 4-0 absorbable monofilament sutures were placed at the other portion of the anastomosis. During the suturing, both stumps were opened to recognize the lumens (Figure 2a). During the ligation, both stumps were closed by moving the peripheral lung tissue to avoid tension at the ligature points (Figure 2b). Interrupted sutures were alternately placed at the cranial and caudal portions to adjust the calibre mismatch between the proximal and distal stumps. After two-thirds of anastomosis was over, few differences remained in the directions of both stumps. The membranous portion of the bronchus was sutured and ligated, and the bronchoplasty was completed. Throughout the bronchoplasty procedure, we sewed the proximal bronchial stump with wide pitches and the distal bronchial stump with narrow pitches to correct the calibre mismatch. Because an intrapulmonary bronchus does not have a cartilaginous ring, the distal stump was expanded to fit the proximal stump. We sometimes used large bites to avoid cutting the bronchial walls. When the bronchial walls were fragile, ligation was started at a portion with decreased tension after all sutures were completed. The anastomotic sites were covered circumferentially with a pedicled pericardial fat pad.

Induction chemoradiotherapy with platinum-based doublet and concurrent radiotherapy (40 Gy) was generally planned for patients with enlarged, but completely removable, N2 lymph node

a

b

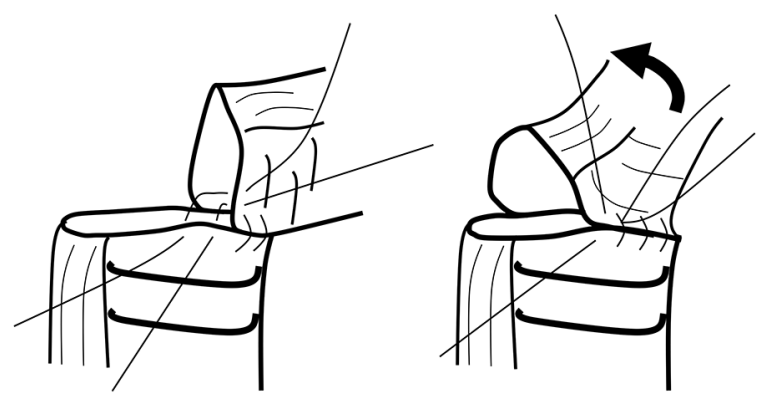

Figure 2. (a) During suturing, both stumps were opened to recognize the lumens. (b) During ligation, both stumps were closed by moving the peripheral lung tissue to avoid tension at the ligature points.

metastasis; however, it was not mandatory for all patients with N2 lymph node metastasis. Patients with pathological stage II and III lung cancer received adjuvant platinum-based doublet chemotherapy, whereas those with stage I lung cancer received oraltegafur adjuvant chemotherapy. We did not set criteria for omitting adjuvant treatment, such treatment being initiated at the discretion of the physician in charge of each case.

After discharge, all patients underwent follow-up chest radiographs and measurement of tumour markers every 2 to 4 months and CT scans at 6 months and every year thereafter. The last follow-up review was conducted on 30 June 2021.

Comorbidities were defined as disorders being treated at the time of diagnosis of lung cancer. A standard bronchoscopic examination was performed 1 week postoperatively. When wound healing at the anastomosis site seemed protracted, the bronchoscopic examination was repeated until healing occurred. When chest radiography revealed an abnormality, the patient underwent a CT examination. Adverse events within 3 months postoperatively were evaluated according to the Common Terminology Criteria for Adverse Events (version 5.0). Adverse events of grade $\geq 3$ were recorded.

\section{Results}

This study included 17 patients. Right middle and lower sleeve lobectomy was performed in five patients, left lower sleeve lobectomy was performed in seven, and left lower and lingular segment sleeve lobectomy was performed in five. Details of the patients' characteristics are shown in Table I. One patient with left lower lung cancer massively infiltrating the left main bronchus was treated with induction chemoradiotherapy to avoid pneumonectomy. Another patient developed a tumour in the right lower lobe with mass formation in the left atrium after definitive treatment by platinum doublet chemotherapy with immunotherapy. He underwent right middle and lower sleeve lobectomy with combined resection of the left atrium.

Neither treatment-related death nor hospitalization-related deaths occurred. Table II shows the details of adverse events after Y-sleeve lobectomy. One patient developed a bronchopleural fistula (BPF) after anastomosis between the 
Table I. Characteristics of patients included in this study.

\begin{tabular}{llc}
\hline Age (years) & & $71(48-82)$ \\
Gender & Male/Female & $13 / 4$ \\
Smoking history & Yes/No & $17 / 0$ \\
Comorbidities & Cardiovascular disease & 8 \\
& Cerebrovascular disease & 3 \\
& Diabetes mellitus & 2 \\
& Other organ malignant tumor* & 1 \\
& Liver disease & 1 \\
Clinical T factor & $1 / 2 / 3 / 4$ & $4 / 5 / 4 / 4$ \\
Clinical N factor & O/1/2 & $5 / 9 / 3$ \\
Clinical Stage & I/II/III & $4 / 5 / 8$ \\
Surgical procedure & Right middle and lower & 5 \\
& sleeve bilobectoy & \\
& Left lower sleeve lobectomy & 7 \\
& Left lower and lingular & 5 \\
Histological subtypes & segment sleeve lobectomy & \\
& Squamous cell carcinoma & 14 \\
& Adenocarcinoma & 1 \\
& Pleomorphic carcinoma & 1 \\
Additional resection & Small cell carcinoma & 1 \\
& Pulmonary artery & 4 \\
& Left atrium & 2 \\
Pathological T factor** & Esophageal muscle & 2 \\
Pathological N factor** & Vagus nerve & 2 \\
Adjuvant chemotherapy & 1/2/3/4 & $4 / 5 / 2 / 5$ \\
& I/II/III & $3 / 6 / 7$ \\
& & $2 / 4 / 10$ \\
& & $7(41 \%)$ \\
\hline
\end{tabular}

*Malignant tumor within five years. **A patient without residual tumor after preoperative treatment was excluded.

left main and upper segment bronchi. The BPF was caused by an ischaemic change in the proximal stump. The patient was treated with induction chemoradiotherapy. The proximal stump was too close to the infiltrated tumour, which disappeared after chemoradiotherapy. He underwent a completion pneumonectomy. Four patients developed pneumonia and were cured by repeated bronchoscopies and antibiotic therapy. Three patients had retention of pleural effusion and two had prolonged air leakage. One patient had empyema after prolonged air leakage and was cured by thoracic drainage and antibiotic therapy.

Because of tumour infiltration, the most peripheral pulmonary artery branch to the preserved lung was removed in two of five patients who underwent right middle and lower sleeve lobectomy and in two of five patients who underwent left lower and lingular segment sleeve lobectomy. No airway complications were observed these four patients after bronchoplasty.

\section{Discussion}

We showed our surgical techniques and analysed short-term outcomes of Y-sleeve lobectomy. The proximal stump is
Table II. Details of adverse events after Y-sleeve lobectomy.

\begin{tabular}{lccc}
\hline & $\begin{array}{c}\text { RMLL } \\
\text { sleeve } \\
\text { lobectomy } \\
(\mathrm{n}=5)\end{array}$ & $\begin{array}{c}\text { LLL } \\
\text { sleeve } \\
\text { lobectomy } \\
(\mathrm{n}=7)\end{array}$ & $\begin{array}{c}\text { LLL and lingular } \\
\text { segment sleeve } \\
\text { lobectomy } \\
(\mathrm{n}=5)^{*}\end{array}$ \\
\hline $\begin{array}{l}\text { Pleural effusion } \\
\text { Pneumonia }\end{array}$ & 1 & 1 & 2 \\
$\begin{array}{l}\text { Empyema } \\
\text { Prolonged air leakage }\end{array}$ & 1 & & 2 \\
$\begin{array}{l}\text { Broncho-pleural fistula } \\
\text { Brain infarction }\end{array}$ & & 1 & 1 \\
Paralytic ileus & & & 1 \\
\hline
\end{tabular}

RMLL, Right middle and lower lobe; LLL, left lower lobe. *Some patients had more than one adverse events.

always fixed in the mediastinum and the distal stump is fixed by surrounding structures such as the pulmonary artery and vein. Significant differences in the directions of the proximal and distal stumps were observed after Y-sleeve lobectomy. These differences may increase the difficulty of Y-sleeve lobectomy. The details of Y-sleeve lobectomy have been investigated in only one other study to date (3). In that study, the rate of major complications was $10.7 \%$. The study included 28 patients who underwent Y-sleeve lobectomy from 1989 to 2015 . The operation-related morbidity and mortality rates after bronchoplasty were significantly better in current cases than in past cases (4). Our study included only current cases and thus more accurately revealed the recent outcomes of Y-sleeve lobectomy.

In our series, one patient developed a BPF after Y-sleeve lobectomy. The cause of the BPF might have been ischaemic change in the proximal stump after induction chemoradiotherapy. Patients undergoing induction mediastinal radiotherapy are reportedly at higher risk of bronchial complications (5). Close attention should be paid to the possibility of a postoperative BPF after induction chemoradiotherapy. Large calibre mismatch is usually observed in bronchoplasty after right middle and lower or left lower lobe and lingular segment sleeve lobectomy, and it sometimes causes retention of secretions followed by pneumonia (2). Because the preserved lung tissues are not massive enough to occupy the residual thoracic cavity, retention of pleural effusion and prolonged air leakage are sometimes observed. Kinking of the left superior pulmonary vein after Y-sleeve lobectomy has been reported (6). We routinely check the deflection of vessels when the preserved lung is inflated. Following the completion of bronchoplasty in the present study, the pericardial fat pad was inserted between the left superior pulmonary vein and anastomosis site. This fat pad helped decrease deflection and prevent kinking of the left superior pulmonary vein. We did not 
observe such complications in the present study. Sleeve lobectomy is superior to pneumonectomy with respect to prognosis $(7,8)$, preservation of pulmonary function $(9)$, and postoperative quality of life (10). Sleeve lobectomy is recommended if anatomically appropriate, and it can allow for margin-negative resection. Favorable prognoses and local controllability of left lower lobe and lingular segment sleeve lobectomy (11) as well as right middle and lower or left lower sleeve lobectomy (3) was reported previously. Only one patient developed a severe adverse event (BPF). No treatment-related deaths occurred. We consider Y-sleeve lobectomy to be a reliable surgical method with which to avoid pneumonectomy.

In contrast to other types of airway reconstruction, tension at the anastomotic site is less severe at the beginning of anastomosis after Y-sleeve lobectomy. After the ventral portion was fixed, there was relatively severe tension at the cranial and caudal portions of the anastomotic site because of the different directions of the distal and proximal stumps. Both stumps were closed by moving peripheral lung tissue to avoid tension at the ligation points. We prefer the open approach rather than a video-assisted procedure because the position of the peripheral lung tissue during bronchoplasty is important to avoid complications in this type of airway reconstruction.

In the present study, we sacrificed the most peripheral pulmonary artery branch to achieve complete resection in four patients, and they developed no airway complications. After the sleeve bronchial resection, the blood supply of the distal stump was provided by the pulmonary artery (12). Toyooka et al. (13) reported that removing the pulmonary artery branch to the preserved lung was a risk factor for BPF in sleeve lobectomy after chemoradiotherapy. Patients undergoing induction mediastinal radiotherapy are reportedly at higher risk of bronchial complications (5). In patients who did not receive induction chemoradiotherapy, sacrificing the pulmonary artery branch may be acceptable to achieve complete resection. The healing processes after airway reconstruction are mainly associated with the blood supply and tension at the anastomosis site. Whether to sacrifice the pulmonary artery branch depends on its size and the tension persisting at the anastomosis site.

This study had certain limitations. First, it was a small retrospective study. Accumulation of data from more patients and further analyses are now ongoing. Second, long-term outcomes were not investigated. Y-sleeve lobectomy is expected to provide oncological benefits similar to those provided by other types of sleeve lobectomy. We will be able to show long-term outcomes after a sufficient follow-up period. Finally, treatments were selected at the discretion of the physician in charge of each case. Selection criteria for surgical procedures should be established in further studies.

In conclusion, we herein presented our surgical techniques and analysed short-term outcomes of Y-sleeve lobectomy.
The appropriate position of the peripheral lung tissue during bronchoplasty is essential to successfully complete the anastomosis. A severe adverse event was observed only in one patient. No treatment-related deaths occurred. Y-sleeve lobectomy is suggested to be a reliable surgical method to avoid pneumonectomy.

\section{Conflicts of Interest}

The Authors have no conflicts of interest to declare regarding this study.

\section{Authors' Contributions}

Takuma Tsukioka designed this study, analysed the data, prepared the figures, and wrote the original draft. Nobuhiro Izumi and Noritoshi Nishiyama oversaw the study and revised the article. All Authors reviewed the article.

\section{References}

1 Palade E, Holdt $\mathrm{H}$ and Passlick B: Bronchus anastomosis after sleeve resection for lung cancer: does the suture technique have an impact on postoperative complication rate? Interact Cardiovasc Thorac Surg 20(6): 798-804, 2015. PMID: 25810291. DOI: $10.1093 /$ icvts/ivv058

2 Tsukioka T, Izumi N, Komatsu H, Inoue H, Miyamoto H, Ito R, Kimura $\mathrm{T}$ and Nishiyama N: Influence of caliber mismatch on patients' clinical course after sleeve lobectomy. Gen Thorac Cardiovasc Surg 69(7): 1079-1085, 2021. PMID: 33507486. DOI: $10.1007 / \mathrm{s} 11748-020-01582-1$

3 Maurizi G, Ciccone AM, Vanni C, D’Andrilli A, Ibrahim M, Andreetti C, Menna C, Tierno SM, Venuta F and Rendina EA: Reimplantation of the upper lobe bronchus after lower sleeve lobectomy or bilobectomy: long-term results. Eur J Cardiothorac Surg 53(6): 1180-1185, 2018. PMID: 29360965. DOI: 10.1093/ ejcts/ezx494

4 Nagayasu T, Yamasaki N, Tsuchiya T, Matsumoto K, Miyazaki $\mathrm{T}$, Hatachi G, Watanabe $\mathrm{H}$ and Tomoshige K: The evolution of bronchoplasty and broncho-angioplasty as treatments for lung cancer: evaluation of 30 years of data from a single institution. Eur J Cardiothorac Surg 49(1): 300-306, 2016. PMID: 25721822. DOI: $10.1093 /$ jets/ezv065

5 Comacchio GM, Schiavon M, Azzolina D, Mammana M, Marulli G, Zuin A, Verderi E, Monaci N, Bonanno L, Pasello G and Rea F: Does induction therapy increase anastomotic complications in bronchial sleeve resections? World J Surg 43(5): 1385-1392, 2019. PMID: 30659342. DOI: 10.1007/ s00268-019-04908-0

6 Ugalde PA, Gregorie J, Zhinguang Y, Boultifat R and Deslauriers J: Left lower lobe sleeve resection. In: Thoracic Surgery. Lung Resections, Bronchoplasty. Mathisen DJ, Morse CR (eds). Philadelphia, Wolters Kluwer, pp. 391-394, 2015.

7 Pagès $\mathrm{PB}$, Mordant $\mathrm{P}$, Renaud S, Brouchet L, Thomas PA, Dahan M, Bernard A and Epithor Project (French Society of Thoracic and Cardiovascular Surgery): Sleeve lobectomy may provide better outcomes than pneumonectomy for non-small cell lung cancer. A decade in a nationwide study. J Thorac 
Cardiovasc Surg 153(1): 184-195.e3, 2017. PMID: 27814899. DOI: $10.1016 / j$.jtcvs.2016.09.060

8 Abdelsattar ZM, Shen KR, Yendamuri S, Cassivi S, Nichols FC 3rd, Wigle DA, Allen MS and Blackmon SH: Outcomes after sleeve lung resections versus pneumonectomy in the United States. Ann Thorac Surg 104(5): 1656-1664, 2017. PMID: 28935348. DOI: 10.1016/j.athoracsur.2017.05.086

9 Gómez-Caro A, Garcia S, Reguart N, Cladellas E, Arguis P, Sanchez M and Gimferrer JM: Determining the appropriate sleeve lobectomy versus pneumonectomy ratio in central nonsmall cell lung cancer patients: an audit of an aggressive policy of pneumonectomy avoidance. Eur J Cardiothorac Surg 39(3): 352-359, 2011. PMID: 21185734. DOI: 10.1016/j.ejcts. 2010.07.002

10 Balduyck B, Hendriks J, Lauwers P and Van Schil P: Quality of life after lung cancer surgery: a prospective pilot study comparing bronchial sleeve lobectomy with pneumonectomy. J Thorac Oncol 3(6): 604-608, 2008. PMID: 18520798. DOI: $10.1097 / J T O .0 b 013 \mathrm{e} 318170 \mathrm{fca} 4$
11 Hishida T, Aokage $\mathrm{K}$, Yoshida J, Miyoshi $\mathrm{T}$ and Tsuboi M: Extended bronchoplasty for locally advanced left lower lobe lung cancer: surgical technique and outcomes. Interact Cardiovasc Thorac Surg 27(4): 602-605, 2018. PMID: 29617785. DOI: $10.1093 /$ icvts/ivy081

12 Ishihara T, Nemoto E, Kikuchi K, Kato R and Kobayashi K: Does pleural bronchial wrapping improve wound healing in right sleeve lobectomy? J Thorac Cardiovasc Surg 89(5): 665-672, 1985. PMID: 3990318.

13 Toyooka S, Soh J, Shien K, Sugimoto S, Yamane M, Oto T, Date $\mathrm{H}$ and Miyoshi S: Sacrificing the pulmonary arterial branch to the spared lobe is a risk factor of bronchopleural fistula in sleeve lobectomy after chemoradiotherapy. Eur J Cardiothorac Surg 43(3): 568-572, 2013. PMID: 22659891. DOI: 10.1093/ejcts/ezs323

Received October 4, 2021

Revised October 27, 2021

Accepted November 18, 2021 